\title{
QT Interval Prolongation in COVID-19: Hypercytokinaemia is the Forgotten Culprit and Hydroxychloroquine Simply Add Fuel to the Fire
}

\author{
Sudhir Mehta ${ }^{1 *}$, Sudhir Bhandari ${ }^{2}$ and Shaurya Mehta \\ ${ }^{1}$ SMS Medical College (University Medical School), Jaipur, India \\ 2Jaslok Hospital \& Research Centre, Mumbai, India
}

Accumulating data suggest an increased cardiovascular disease morbidity and mortality in Covid-19 patients [1]. In particular, growing evidence suggest that COVID-19 has a higher risk of arrhythmic events, with important implications for survival [1].

The prevalence of arrhythmias varies in COVID-19. In a study of 138 patients from Wuhan, who were hospitalized with COVID-19 related pneumonia, arrhythmias were reported in $17 \%$ of the general cohort and in $44 \%$ of patients admitted to an intensive care unit [2]. About $30 \%$ of patients with COVID-19 have myocardial injury [3] which predisposes to conduction abnormalities including prolonged QT interval. However, in intensive care unit patients, despite the high frequency of arrhythmias ( $\approx 50 \%$ of cases), only half have acute cardiac injury which suggests that factors other than myocardial damage are also involved in enhancing the arrhythmic risk in COVID-19.

A subgroup of patients with severe COVID-19 has a cytokine storm syndrome. Secondary Haemophagocytic lymphohistiocytosis (sHLH) is an under-recognized, hyper-inflammatory syndrome characterized by a fulminant and fatal hypercytokinaemia with multi-organ failure. In adults, $\mathrm{sHLH}$ is most commonly triggered by viral infections [4] and occurs in 3.7$4.3 \%$ of sepsis cases [5]. A cytokine profile resembling $\mathrm{SHLH}$ is associated with COVID-19 disease severity, characterized by increased interleukin (IL)-2, IL-7, granulocyte colony stimulating factor, interferon- $\gamma$ inducible protein 10 , monocyte chemo-attractant protein 1 , macrophage inflammatory protein $1-\alpha$, and tumor necrosis factor- $\alpha$ [6].

Excess levels of pro-inflammatory cytokines may play a role in causing prolongation of the QT interval [7]. It has been demonstrated that IL-6, tumor necrosis factor (TNF) $\alpha$, and IL-1 can prolong ventricular action potential duration by modulating the expression or function of several cardiomyocyte ion channels, specifically $\mathrm{K}+$ and $\mathrm{Ca} 2+$ channels (inflammatory cardiac channelopathies) [8].

It has also been observed that patients with rheumatoid arthritis (RA) have a markedly increased incidence of QT prolongation and an increased mortality due to cardiac arrhyth- mias [9]. Patients with RA have twice the risk of death from ventricular arrhythmias as compared to the normal person, and a 50 ms increase in QT in patients with RA doubles the risk for all-cause mortality [9]. This increase in mortality risk also correlates with increases in the levels of inflammatory markers [9].

In a study, Lazzerini, et al. [10] noted that patients with RA treated with tocilizumab, an IL6 blocker, had shortening of their previously prolonged QT interval, and this shortening was associated with concomitant decreases in inflammatory markers.

Haberman R, et al. [11] showed that the baseline use of biologics (anticytokine and other immunosuppressives) was not associated with worse Covid-19 outcomes in 86 established patients of immune-mediated inflammatory diseases at New York University Langone Health in New York City. In other words, Covid-19 patients on immunosuppressives, despite having higher risk, fared better, suggesting that these therapies minimized the effects of cytokine release in Covid-19 disease.

Data on hydroxychloroquine (HCQ) suggest it has a low risk of causing Torsades de Pointes (TdP), based on its use in rheumatoid arthritis, systemic lupus erythematosus, and anti-malarial therapy [12]. Torsade de pointes is a rare event, with an estimated annual crude rate of 3.2 per million population.

Various studies [13-15] have suggested increased risk of QTc prolongation with use of HCQ with/without Azithromycin in Covid-19 patients. However, the risks of cardiac ad-

*Corresponding author: Prof Sudhir Mehta, SMS Medical College (University Medical School), Jaipur, India

Accepted: November 05, 2020

Published online: November 07, 2020

Citation: Mehta S, Bhandari S, Mehta S (2020) QT Interval Prolongation in COVID-19: Hypercytokinaemia is the Forgotten Culprit and Hydroxychloroquine Simply Add Fuel to the Fire. Arch Immunol 2(1):22-23 
Citation: Mehta S, Bhandari S, Mehta S (2020) QT Interval Prolongation in COVID-19: Hypercytokinaemia is the Forgotten Culprit and Hydroxychloroquine Simply Add Fuel to the Fire. Arch Immunol 2(1):22-23

verse events associated with HCQ are amplified in COVID-19 for several reasons [16]. Patients with SARS-CoV-2 infection have multiple risk factors for drug-induced proarrhythmia-hypokalemia, hypoxia, underlying cardiovascular disease and concomitant use of QT interval prolonging drugs. Although, QTc prolongation was more commonly seen in hospitalized patients esp. in ICU settings receiving HCQ with/without Azithromycin, none of the studies correlated QTc interval prolongation with cytokine levels.

Based on above observations, further studies need to be undertaken to address this issue: To what extent pro-inflammatory cytokines influence QTc interval prolongation in Covid-19? and will anti-cytokine therapies reduce mortality due to cardiac arrhythmias?

Putting blame solely on $\mathrm{HCQ} /$ Azithromycin on prolongation of QTc interval is not justified. The other risk factors also contribute to cardiac adverse effects. The knowledge gap needs to be answered in well-designed clinical trials.

\section{Authors Declarations}

No conflict of interest.

No funding received for this manuscript.

All authors contributed equally.

\section{References}

1. Driggin E, Madhavan MV, Bikdeli B, et al. (2020) Cardiovascular considerations for patients, health care workers, and health systems during the coronavirus disease 2019 (COVID-19) pandemic. J Am Coll Cardiol 75: 2352-2371.

2. Wang D, Hu B, Hu C, et al. (2020) Clinical Characteristics of 138 Hospitalized Patients with 2019 Novel Coronavirus-Infected Pneumonia in Wuhan, China. JAMA 323: 1061-1069.

3. https://doi.org/10.1001/jamacardio.2020.1017

4. Ramos-Casals M, Brito-Zeron P, Lopez-Guillermo A, et al. (2014) Adult haemophagocytic syndrome. Lancet 383: 1503-1516.

5. Karakike E, Giamarellos-Bourboulis EJ (2019) Macrophage activation-like syndrome: A distinct entity leading to early death in sepsis. Front Immunol 10: 55.
6. Huang C, Wang Y, Li X, et al. (2020) Clinical features of patients infected with 2019 novel coronavirus in Wuhan, China. Lancet 395: 497-506.

7. Lazzerini PE, Capecchi PL, Laghi-Pasini F (2017) Systemic inflammation and arrhythmic risk: Lessons from rheumatoid arthritis. Eur Heart J 38: 1717-1727.

8. Lazzerini PE, Laghi-Pasini F, Boutjdir M, et al. (2019) Cardioimmunology of arrhythmias: The role of autoimmune and inflammatory cardiac channelopathies. Nat Rev Immunol 19: 63-64.

9. Panoulas VF, Toms TE, Douglas KM, et al. (2014) Prolonged QTc interval predicts all-cause mortality in patients with rheumatoid arthritis: An association driven by high inflammatory burden. Rheumatology 53: 131-137.

10. Lazzerini PE, Acampa M, Capecchi PL, et al. (2015) Antiarrhythmic potential of anticytokine therapy in rheumatoid arthritis: Tocilizumab reduces corrected QT interval by controlling systemic inflammation. Arthritis Care Res 67: 332-339.

11. Haberman R, Axelrad J, Chen A, et al. (2020) Covid-19 in immune-mediated inflammatory diseases-case series from New York. N Engl J Med 383: 85-88.

12. Haeusler IL, Chan XHS, Guérin PJ, et al. (2018) The arrhythmogenic cardiotoxicity of the quinoline and structurally related antimalarial drugs: A systematic review. BMC Med 16: 200.

13. Mercuro N, Yen CF, Shim D, et al. (2020) Risk of QT Interval Prolongation Associated With Use of Hydroxychloroquine With or Without Concomitant Azithromycin Among Hospitalized Patients Testing Positive for Coronavirus Disease 2019 (COVID-19). JAMA Cardiol 5: 1036-1041.

14. https://doi.org/10.1101/2020.04.02.20047050

15. Bonow R, Hernandez AF, Turakhia M (2020) Hydroxychloroquine, Coronavirus Disease 2019, and QT Prolongation. JAMA Cardiol 5: 986-987.

16. Funck-Brentano C, Salem JE, Nguyen LS, et al. (2020) Response to the editorial "COVID-19 in patients with cardiovascular diseases": COVID-19 treatment with hydroxychloroquine or chloroquine and azithromycin: A potential risk of torsades de pointes. Arch Cardiovasc Dis 113: 367-368.

DOI: $10.36959 / 885 / 369$ 